\title{
Article
}

\section{Analytical Solutions of Viscoelastic Nonlocal Timoshenko Beams}

\author{
Francesco Paolo Pinnola ${ }^{1}$ D, Raffaele Barretta ${ }^{1, *(D)}$, Francesco Marotti de Sciarra ${ }^{1}$ and Antonina Pirrotta ${ }^{2}$ (D) \\ 1 Department of Structures for Engineering and Architecture, University of Naples Federico II, Via Claudio 21, \\ Ed. 6, 80125 Naples, Italy; francescopaolo.pinnola@unina.it (F.P.P.); marotti@unina.it (F.M.d.S.) \\ 2 Department of Engineering, University of Palermo, Via E. Basile, 90128 Palermo, Italy; \\ antonina.pirrotta@unipa.it \\ * Correspondence: rabarret@unina.it
}

check for updates

Citation: Pinnola, F.P.; Barretta, R.; Marotti de Sciarra, F.; Pirrotta, A. Analytical Solutions of Viscoelastic Nonlocal Timoshenko Beams. Mathematics 2022, 10, 477. https:// doi.org/10.3390/math10030477

Academic Editors: Krzysztof Kamil Żur, Jinseok Kim and J. N. Reddy

Received: 29 December 2021

Accepted: 28 January 2022

Published: 1 February 2022

Publisher's Note: MDPI stays neutral with regard to jurisdictional claims in published maps and institutional affiliations.

Copyright: (c) 2022 by the authors. Licensee MDPI, Basel, Switzerland. This article is an open access article distributed under the terms and conditions of the Creative Commons Attribution (CC BY) license (https:// creativecommons.org/licenses/by/ $4.0 /)$.

\begin{abstract}
A consistent nonlocal viscoelastic beam model is proposed in this paper. Specifically, a Timoshenko bending problem, where size- and time-dependent effects cannot be neglected, is investigated. In order to inspect scale phenomena, a stress-driven nonlocal formulation is used, whereas to simulate time-dependent effects, fractional linear viscoelasticity is considered. These two approaches are adopted to develop a new Timoshenko bending model. Analytical solutions and application samples of the proposed formulation are presented. Moreover, in order to show influences of viscoelastic and size effects on mechanical response, parametric analyses are provided. The contributed results can be useful for the design and optimization of small-scale devices exhibiting flexural behaviour.
\end{abstract}

Keywords: MEMS/NEMS; fractional calculus; stress-driven nonlocality; small-scale beams; size effects

\section{Introduction}

Some devices designed at small scales [1-4], such as nano- and micro-sensors, actuators, piezoelectric systems, and AFM indenters can be modeled as beams but require specific stress-strain relations to predict their mechanical behaviour. In this context, the paper proposes a stress-strain relation that is enriched with respect to the classic local elastic one. The presented model is able to model two specific phenomena that occur in the mechanics of several small-scale devices, i.e., size effects and hereditariness.

Size effects can invalidate the hypothesis of the locality of classical continuum mechanical models [5-11]. In this paper, this issue is taken into account and it is overcome with the aid of a nonlocal formulation. Following this approach, the nonlocal strain (or stress) at a continuum point depends on a stress (or strain) field in the whole domain [12,13]. For the problem at hand, the nonlocality is mainly related to the small sizes of the structures and the influences of the stress-strain relation on its space-dependence. Instead, the other considered phenomenon arises when the material that builds the small-sized structure exhibits a time-dependent viscoelastic stress-strain relation [14,15]. Both phenomena are modeled by a stress-driven integral formulation where the nonlocal effect is modeled by a Fredholm integral, in which the kernel is a space attenuation function that weights the long-range mechanical interactions [16], whereas the viscoelastic phenomenon is given by a Boltzmann convolution integral with a time-dependent causal kernel that rules the memory effects [17].

There are various ways to model nonlocalities [18-25], among these approaches the adopted formulation is derived from Eringen's theory [26,27]. In such a model, the nonlocal stress is expressed by the convolution integral between an averaging kernel and the local stress field. However, some paradoxes and mathematical issues appear in the original strain-driven Eringen's formulation for real bounded systems [28-30]. The simplest way to overcome these issues is obtained by the integral stress-driven approach [31,32]. While in the original Eringen's formulation the input is the deformation and the output is the stress, in the stress-driven formulation, nonlocal strain and stress have reversed roles while 
maintaining the same type of constitutive relationship. That consistent approach [31,32], useful also to detect some analytical solutions of mechanical problems of applicative interest [33], is adopted in this paper to formulate a proper nonlocal stress-strain relation. In this way the strain at a point becomes a function of the stress field introducing a space dependence that makes the nonlocal constitutive law different from the local elastic one.

Another difference of the considered stress-strain relation from the classical elastic one is related to the time-dependence due to viscoelastic phenomena. This produces another nonlocal effect that differs from the previous one because it manifests itself over time. It implies that the mechanical state of the past influences all responses in the future. Materials that possess this mechanical memory are called hereditary, and for them stress decays under constant strain, and strain grows up under constant stress, showing a time-dependent mechanical behaviour. Obviously, thetheory of elasticity is not able to reproduce this phenomenon but an approach to overcome this limit is provided by the linear viscoelasticity. For this theory, the mechanical behaviour of viscoelastic/hereditary material is considered intermediate between elastic/solid and viscous/fluid ones. Following this approach, the integral formulation of hereditariness provides a stress-strain relation as an integral Volterra-Boltzmann relation, wherein the kernel is a time-dependent function that takes into account the memory of the material. The choice of the time-dependent convolution kernel yields different kinds of viscoelastic models. For their capability to fit experimental data, in this paper, we consider a power-law-type kernel that leads to a hereditary model based on fractional calculus. Several works have proven that the fractional viscoelastic model is able to represent the real time-dependent behaviour of a wide variety of materials [34-40]. For this reason it is adopted in this manuscript to obtain a versatile stress-strain relation.

The proposed nonlocal time-dependent stress-strain relation based upon a stressdriven integral formulation and fractional-order operators is applied to study the bending problem of micro- and nano-beams. Specifically, this article shows a useful approach to finding analytical solutions of the viscoelastic nonlocal Timoshenko bending problem. In this way, a mechanical model and a parametric study - useful for mechanical design of advanced structural devices at small scales-are provided, and some useful tools for predicting the time-dependent behaviour of nano-systems are shown in detail.

\section{Fractional Viscoelasticity for Beam Problems}

This section introduces the local stress-strain relation for time-dependent bending problems. The constitutive law of a hereditary material is derived by means of a Boltzmann integral formulation of linear viscoelasticity $[14,15]$. Following this approach and with a proper selection of the time-dependent convolution kernel, the fractional-order stress-strain relation is derived.

\subsection{Stress and Strain in Bending Problems}

Let us consider a 3D, continuous beam element referred to in coordinate Cartesian axes $(x, y, z)$, with $z$ centroidal axis. The viscoelastic stress and strain of the beam are described by time-dependent matrices:

$$
\mathbf{T}=\left[\begin{array}{ccc}
0 & 0 & \tau_{x z} \\
0 & 0 & \tau_{y z} \\
\tau_{z x} & \tau_{z y} & \sigma_{z}
\end{array}\right], \quad \mathbf{E}=\left[\begin{array}{ccc}
\varepsilon_{x} & 0 & \frac{1}{2} \gamma_{x z} \\
0 & \varepsilon_{y} & \frac{1}{2} \gamma_{y z} \\
\frac{1}{2} \gamma_{z x} & \frac{1}{2} \gamma_{z y} & \varepsilon_{z}
\end{array}\right]
$$

where $\mathbf{T}$ is the stress tensor of a plane state and $\mathbf{E}$ is a strain tensor. Diagonal elements of $\mathbf{E}$ can be written as functions of the component $\varepsilon_{z}$, that is, $\varepsilon_{x}=\varepsilon_{y}=-v \varepsilon_{z}$, where $v$ is the Poisson's ratio. Both the tensors above can be decomposed in spherical and deviatoric parts, as follows:

$$
\mathbf{T}=\mathbf{T}_{\mathbf{s}}+\mathbf{T}_{\mathbf{d}}=\frac{1}{3} \sigma_{z} \mathbf{I}+\left[\begin{array}{ccc}
-\frac{1}{3} \sigma_{z} & 0 & \tau_{x z} \\
0 & -\frac{1}{3} \sigma_{z} & \tau_{y z} \\
\tau_{z x} & \tau_{z y} & \frac{2}{3} \sigma_{z}
\end{array}\right]
$$




$$
\mathbf{E}=\mathbf{E}_{\mathbf{s}}+\mathbf{E}_{\mathbf{d}}=\frac{1-2 v}{3} \varepsilon_{z} \mathbf{I}+\left[\begin{array}{ccc}
-\frac{1+v}{3} \varepsilon_{z} & 0 & \frac{1}{2} \gamma_{x z} \\
0 & -\frac{1+v}{3} \varepsilon_{z} & \frac{1}{2} \gamma_{y z} \\
\frac{1}{2} \gamma_{z x} & \frac{1}{2} \gamma_{z y} & \frac{2(1+v)}{3} \varepsilon_{z}
\end{array}\right],
$$

where $\mathbf{I}$ is the identity tensor.

The beam is made of homogeneous viscoelastic isotropic material, therefore, stress and strain tensors are time-dependent entities related by the Boltzmann integral relation. That is:

$$
\begin{aligned}
& \mathbf{T}_{\mathbf{s}}(t)=\int_{-\infty}^{t} \mathcal{G}_{1}(t-\bar{t}) \partial_{\bar{t}} \mathbf{E}_{\mathbf{s}}(\bar{t}) \mathrm{d} \bar{t}, \\
& \mathbf{T}_{\mathbf{d}}(t)=\int_{-\infty}^{t} \mathcal{G}_{2}(t-\bar{t}) \partial_{\bar{t}} \mathbf{E}_{\mathbf{d}}(\bar{t}) \mathrm{d} \bar{t},
\end{aligned}
$$

where $\mathcal{G}_{1}(t)$ and $\mathcal{G}_{2}(t)$ are independent relaxation functions relative to states of dilatation and of shear, respectively [14,15].

Viscoelastic stress-strain relations are obtained from Equation (3)

$$
\begin{gathered}
\sigma_{z}(t)=\frac{1-2 v}{3} \int_{-\infty}^{t} \mathcal{G}_{1}(t-\bar{t}) \partial_{\bar{t}} \varepsilon_{z}(\bar{t}) \mathrm{d} \bar{t}+\frac{2(1+v)}{3} \int_{-\infty}^{t} \mathcal{G}_{2}(t-\bar{t}) \partial_{\bar{t} \varepsilon_{z}}(\bar{t}) \mathrm{d} \bar{t} \\
\tau_{i z}(t)=\frac{1}{2} \int_{-\infty}^{t} \mathcal{G}_{2}(t-\bar{t}) \partial_{\bar{t}} \gamma_{i z}(\bar{t}) \mathrm{d} \bar{t}, \quad i \in\{x, y\} .
\end{gathered}
$$

Considering that Poisson's ratio is time-independent, we get

$$
\frac{\mathcal{G}_{1}(t)}{\mathcal{G}_{2}(t)}=\frac{1+v}{1-2 v},
$$

and the two relaxation functions are expressed by

$$
\mathcal{G}_{1}(t)=\frac{\mathrm{E}(t)}{1-2 v}, \quad \mathcal{G}_{2}(t)=\frac{\mathrm{E}(t)}{1+v},
$$

with $\mathrm{E}(t)$ axial relaxation modulus. Under these assumptions, Equation (4) yields

$$
\begin{aligned}
\sigma_{z}(t) & =\int_{-\infty}^{t} \mathrm{E}(t-\bar{t}) \partial_{\bar{t}} \varepsilon_{z}(\bar{t}) \mathrm{d} \bar{t}=\varepsilon_{z}(0) \mathrm{E}(t)+\int_{0}^{t} \mathrm{E}(t-\bar{t}) \partial_{\bar{t}} \varepsilon_{z}(\bar{t}) \mathrm{d} \bar{t}, \\
\tau_{i z}(t) & =\int_{-\infty}^{t} \mathrm{G}(t-\bar{t}) \partial_{\bar{t}} \gamma_{i z}(\bar{t}) \mathrm{d} \bar{t}=\gamma_{i z}(0) \mathrm{G}(t)+\int_{0}^{t} \mathrm{G}(t-\bar{t}) \partial_{\bar{t}} \gamma_{i z}(\bar{t}) \mathrm{d} \bar{t},
\end{aligned}
$$

$\mathrm{G}(t)$ is the shear relaxation modulus defined by

$$
\mathrm{G}(t)=\frac{E(t)}{2(1+v)} .
$$

The stress-strain relations in terms of strain components are

$$
\begin{aligned}
\varepsilon_{z}(t) & =\int_{-\infty}^{t} \mathcal{J}_{\mathrm{E}}(t-\bar{t}) \partial_{\bar{t}} \sigma_{z}(\bar{t}) \mathrm{d} \bar{t}=\sigma_{z}(0) \mathcal{J}_{\mathrm{E}}(t)+\int_{0}^{t} \mathcal{J}_{\mathrm{E}}(t-\bar{t}) \partial_{\bar{t}} \sigma_{z}(\bar{t}) \mathrm{d} \bar{t} \\
\gamma_{i z}(t) & =\int_{-\infty}^{t} \mathcal{J}_{\mathrm{G}}(t-\bar{t}) \partial_{\bar{t}} \tau_{i z}(\bar{t}) \mathrm{d} \bar{t}=\tau_{i z}(0) \mathcal{J}_{\mathrm{G}}(t)+\int_{0}^{t} \mathcal{J}_{\mathrm{G}}(t-\bar{t}) \partial_{\bar{t}} \tau_{i z}(\bar{t}) \mathrm{d} \bar{t}
\end{aligned}
$$

The integral kernels $\mathcal{J}_{\mathrm{E}}(t)$ and $\mathcal{J}_{\mathrm{G}}(t)$ are creep compliances, related to relaxation counterparts in Laplace domain by the following relation

$$
\hat{\mathcal{J}}_{\mathrm{E}}(s) \hat{\mathrm{E}}(s)=\hat{\mathcal{J}}_{\mathrm{G}}(s) \hat{\mathrm{G}}(s)=s^{-2},
$$


where $\hat{\imath}$ denotes Laplace transform. Such an integral transform is defined by

$$
\hat{f}(s)=\mathcal{L}\{f(t), s\}:=\int_{-\infty}^{\infty} f(t) e^{-s t} \mathrm{~d} t,
$$

being $s$ a complex number.

\subsection{Fractional-Order Viscoelasticity}

Among the various time-dependent kernels which can be used in the viscoelastic stress-strain relation in Equations (7) and (9) the power-law type is considered in the following to study the nonlocal viscoelastic bending problem. Specifically, we assume that the relaxation modulus are

$$
\mathrm{E}(t)=\frac{\mathrm{E}_{\beta} t^{-\beta}}{\Gamma(1-\beta)}, \quad \mathrm{G}(t)=\frac{\mathrm{G}_{\beta} t^{-\beta}}{\Gamma(1-\beta)}=\frac{\mathrm{E}_{\beta} t^{-\beta}}{2(1+v) \Gamma(1-\beta)}, \quad 0 \leqslant \beta \leqslant 1,
$$

where $E_{\beta}$ and $G_{\beta}$ are time-dependent parameters with dimension $\left[\mathrm{Pas}^{\beta}\right]$. As it is shown by various relaxation tests the order $\beta$ varying between $[0,1]$ for several materials $[34,36,38,39]$.

By placing these relaxation functions into Equation (7) and assuming that $\varepsilon_{z}(0)=$ $\gamma_{i z}(0)=0$, the following relations hold true

$$
\begin{gathered}
\sigma_{z}(t)=\mathrm{E}_{\beta}\left(\mathrm{D}_{0^{+}}^{\beta} \varepsilon_{z}\right)(t), \\
\tau_{i z}(t)=\mathrm{G}_{\beta}\left(\mathrm{D}_{0^{+}}^{\beta} \gamma_{i z}\right)(t)=\frac{\mathrm{E}_{\beta}}{2(1+v)}\left(\mathrm{D}_{0^{+}}^{\beta} \gamma_{i z}\right)(t),
\end{gathered}
$$

where $\Gamma(\cdot)$ is the Euler's gamma function, $\left(\mathrm{D}_{0^{+}}^{\beta} \cdot\right)(t)$ denotes the time-derivative of order $\beta$ with lower bound $t=0$. The stress-strain relation in Equation (13) represents the constitutive law of the so called spring-pot model [35,41-45]. The involved fractional derivative for $t>0$ is

$$
\left(D_{0^{+}}^{\beta} f\right)(t):= \begin{cases}\frac{1}{\Gamma(1-\beta)} \int_{0}^{t}(t-\bar{t})^{1-\beta} \frac{\mathrm{d}}{\mathrm{d} \bar{t}} f(\bar{t}) \mathrm{d} \bar{t} & \text { if } 0 \leqslant \beta<1, \\ \frac{\mathrm{d}}{\mathrm{d} t} f(t) & \text { if } \beta=1\end{cases}
$$

and generalizes the classical integer order derivative [46]. In the next sections, the partial counterpart of the differential operator in Equation (14) will be denoted as $\partial_{t^{+}}^{\beta}$.

By taking into account the relaxation functions in Equation (12) and the relation in Laplace domain in Equation (10) the creep compliances for this fractional model are

$$
\mathcal{J}_{\mathrm{E}}(t)=\frac{t^{\beta}}{\mathrm{E}_{\beta} \Gamma(1+\beta)}, \quad \mathcal{J}_{\mathrm{G}}(t)=\frac{t^{\beta}}{\mathrm{G}_{\beta} \Gamma(1+\beta)}=\frac{2(1+v) t^{\beta}}{\mathrm{E}_{\beta} \Gamma(1+\beta)},
$$

by placing Equation (15) into Equation (9) we obtain

$$
\begin{gathered}
\varepsilon_{z}(t)=\frac{1}{\mathrm{E}_{\beta}}\left(\mathrm{I}_{0^{+}}^{\beta} \sigma_{z}\right)(t), \\
\gamma_{i z}(t)=\frac{1}{\mathrm{G}_{\beta}}\left(\mathrm{I}_{0^{+}}^{\beta} \tau_{i z}\right)(t)=\frac{2(1+v)}{\mathrm{E}_{\beta}}\left(\mathrm{I}_{0^{+}}^{\beta} \tau_{i z}\right)(t),
\end{gathered}
$$

where $\left(\mathrm{I}_{0^{+}}^{\beta} \cdot\right)(t)$ denotes the $\beta$-order Riemann-Liouville fractional integral with lower bound $t=0$ defined by

$$
\left(\mathrm{I}_{t^{+}}^{\beta} f\right)(t):=\frac{1}{\Gamma(\beta)} \int_{0}^{t}(t-\bar{t})^{\beta-1} f(\bar{t}) \mathrm{d} \bar{t},
$$


which generalizes the Cauchy formula for non-integer order $\beta$.

The fractional constitutive laws in Equations (13) and (16) are the generalization of the classical elastic and viscous models. Specifically, the fractional-order relations become the Young elastic laws for $\beta=0$ and the Newton-Petroff viscous law for $\beta=1$. The fractional-order stress-strain relation is able to describe the real time-dependent behaviour of several viscoelastic material by the estimation of a few number of mechanical parameters, i.e., $E_{\beta}, \beta$ and $v$.

\section{Nonlocal Viscoelastic Timoshenko Beam}

This section deals with the static problem of a viscoelastic nonlocal beam under the kinematic assumptions of Timoshenko's theory. Time-dependent behaviour is simulated with the aid of the fractional-order model introduced in the previous section, and nonlocal phenomena are described by an integral stress-driven formulation.

\subsection{Fractional Viscoelastic Beam Model}

Let us consider a stubby beam of length $L$ and cross-section $A$ depicted in Figure 1. Its domain is described with respect to a Cartesian coordinate system $(x, y, z)$, where $x$ and $y$ are principal inertia axes of the cross-section and $z$ is the centroidal longitudinal axis. The beam is forced by the time-dependent transversal load $q_{y}(z, t)$ and spatially distributed moments $m_{x}(z, t)$.
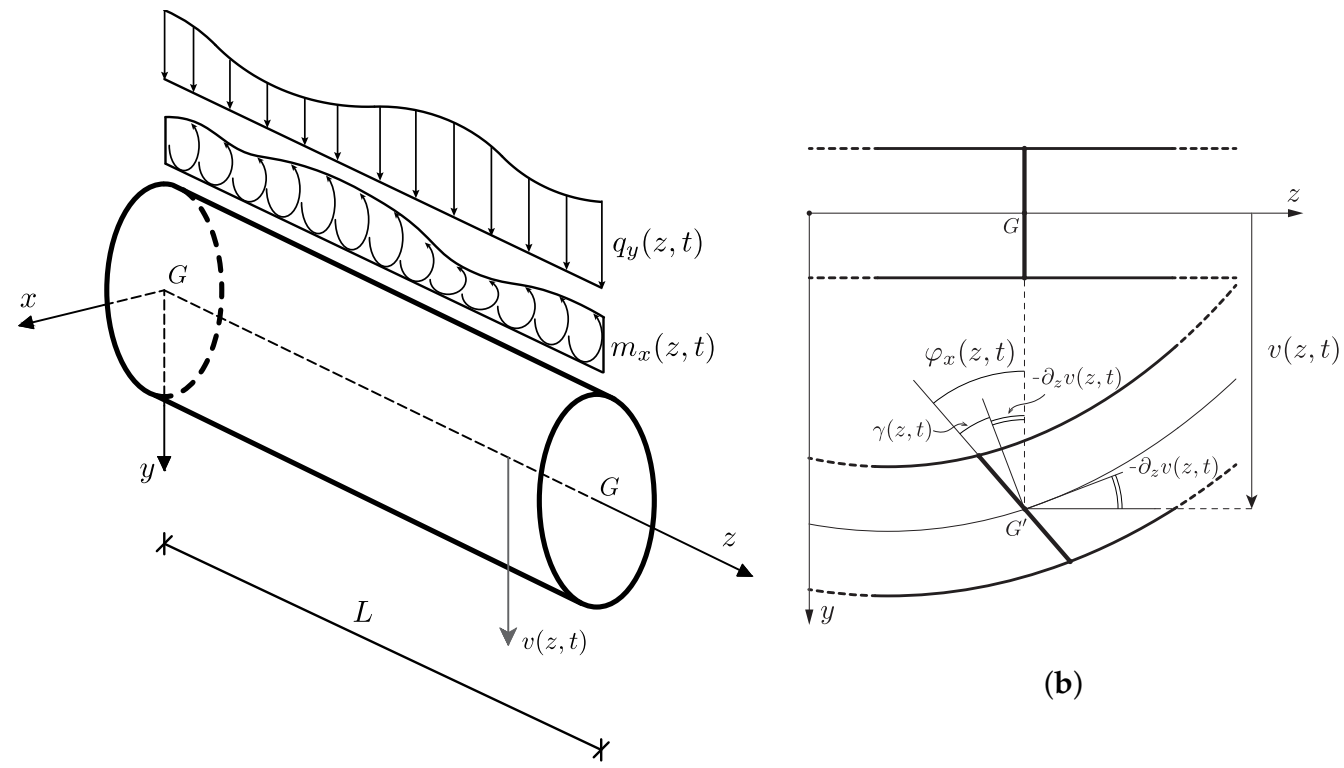

(b)

(a)

Figure 1. Stubby beam. (a) Layout of the beam; (b) kinematic model.

As it is shown in the Figure 1a, the beam is loaded only in the plane $y-z$, therefore, equilibrium is ruled by the following two differential equations

$$
\begin{gathered}
\partial_{z} T_{y}(z, t)+q_{y}(z, t)=0, \\
\partial_{z} M_{x}(z, t)+m_{x}(z, t)=T_{y}(z, t),
\end{gathered}
$$

where $T_{y}(z, t)$ is the shear force and $M_{x}(z, t)$ is the bending moment of the section at abscissa $z$, defined by

$$
\begin{aligned}
T_{y}(z, t) & =\int_{A} \tau_{y z}(z, t) \mathrm{d} A, \\
M_{x}(z, t) & =\int_{A} y \sigma_{z}(z, t) \mathrm{d} A .
\end{aligned}
$$


The first-order differential equilibrium equations in Equation (18) can be combined to obtain a second-order differential equation. That is,

$$
\partial_{z}^{2} M_{x}(z, t)=-q_{y}(z, t)-\partial_{z} m_{x}(z, t) .
$$

Under the Timoshenko's assumptions, after the deformation any cross-section remains plane but its flat does not remain orthogonal to the centerline of the deformed beam. This kinematic model is shown in Figure $1 \mathrm{~b}$ and implies that

$$
\varphi_{x}(z, t)=\gamma(z, t)-\partial_{z} v(z, t),
$$

where $\varphi_{x}(z, t)$ is the rotation of the cross-section around the axis $x, \gamma(z, t)$ is the shear strain, and $v(z, t)$ is the transversal displacement.

Fractional stress-strain relations in Equation (16) and kinematic model assumptions lead to local viscoelastic relations in terms of shear strain and force $\gamma^{\mathrm{ve}}(z, t)-T_{y}(z, t)$ and the bending curvature and moment $\chi_{x}^{\mathrm{ve}}(z, t)-M_{x}(z, t)$. That is,

$$
\begin{aligned}
& \chi_{x}^{\mathrm{ve}}(z, t)=\frac{1}{\mathrm{E}_{\beta} \mathcal{I}_{x} \Gamma(\beta)} \int_{0}^{t}(t-\bar{t})^{\beta-1} M_{x}(z, \bar{t}) \mathrm{d} \bar{t}, \\
& \gamma^{\mathrm{ve}}(z, t)=\frac{1}{\mathrm{G}_{\beta} A_{s} \Gamma(\beta)} \int_{0}^{t}(t-\bar{t})^{\beta-1} T_{y}(z, \bar{t}) \mathrm{d} \bar{t},
\end{aligned}
$$

where $A_{s}=k A$ is the shear area defined as the product of the cross-area and the shear factor $k$, and $\mathcal{I}_{x}$ is defined as

$$
\mathcal{I}_{x}=\int_{A} y^{2} \mathrm{~d} A,
$$

and represents the moment of inertia of the cross-section with respect to the $x$ axis. These local hereditary relations are able to take into account hereditary time-dependent property of the viscoelastic beam. In order to derive the nonlocal beam model, these constitutive relations are used in the next section to obtain the integral stress-driven formulation of the viscoelastic nonlocal Timoshenko's beam.

\subsection{Viscoelastic Stress-Driven Nonlocal Beam}

The nonlocal stress-driven integral model is based on the assumption that the nonlocal viscoelastic kinematic entities are functions of their local counterparts in Equation (22). These laws are represented by two convolution integrals where the kernel is an attenuation space-function $\phi_{\lambda}(z)$ that weights nonlocal effects by means of a dimensionless parameter $\lambda$. That is,

$$
\begin{aligned}
& \chi_{x}(z, t)=\phi_{\lambda}(z) * \chi_{x}^{\mathrm{ve}}(z, t)=\int_{0}^{L} \phi_{\lambda}(z-\bar{z}) \chi_{x}^{\mathrm{ve}}(\bar{z}, t) \mathrm{d} \bar{z} \\
& \gamma(z, t)=\phi_{\lambda}(z) * \gamma^{\mathrm{ve}}(z, t)=\int_{0}^{L} \phi_{\lambda}(z-\bar{z}) \gamma^{\mathrm{ve}}(\bar{z}, t) \mathrm{d} \bar{z} .
\end{aligned}
$$

The local viscoelastic curvature and shear strain are defined in Equation (22), therefore, Equation (24) become

$$
\begin{gathered}
\chi_{x}(z, t)=\int_{0}^{L} \frac{\phi_{\lambda}(z-\bar{z})}{\mathrm{E}_{\beta} \mathcal{I}_{x} \Gamma(\beta)} \int_{0}^{t}(t-\bar{t})^{\beta-1} M_{x}(\bar{z}, \bar{t}) \mathrm{d} \bar{t} \mathrm{~d} \bar{z}, \\
\gamma(z, t)=\int_{0}^{L} \frac{\phi_{\lambda}(z-\bar{z})}{\mathrm{G}_{\beta} A_{s} \Gamma(\beta)} \int_{0}^{t}(t-\bar{t})^{\beta-1} T_{y}(\bar{z}, \bar{t}) \mathrm{d} \bar{t} \mathrm{~d} \bar{z},
\end{gathered}
$$

these equations represent the stress-driven nonlocal viscoelastic relations for the Timoshenko's beam. The convolution kernels must be selected among the symmetric positive functions with limited impulsivity propriety [27]. It can be chosen from among several 
functions, i.e., exponential, Gaussian, power-law type. Below, we adopt as a convolution kernel the following bi-exponential function

$$
\phi_{\lambda}(z)=\frac{1}{2 l_{\lambda}} \exp \left(-\frac{|z|}{l_{\lambda}}\right)
$$

where $l_{\lambda}=\lambda L$ measures long-range nonlocal interactions. Under these assumptions, the integral relations in Equation (25) are equivalent to the following integrodifferential equations

$$
\begin{aligned}
\partial_{z}^{2} \chi_{x}(z, t)-\frac{\chi_{x}(z, t)}{l_{\lambda}^{2}} & =-\frac{1}{l_{\lambda}^{2} \mathrm{E}_{\beta} \mathcal{I}_{x} \Gamma(\beta)} \int_{-\infty}^{t}(t-\bar{t})^{\beta-1} M_{x}(z, \bar{t}) \mathrm{d} \bar{t}, \\
\partial_{z}^{2} \gamma(z, t)-\frac{\gamma(z, t)}{l_{\lambda}^{2}} & =-\frac{1}{l_{\lambda}^{2} \mathrm{G}_{\beta} A_{s} \Gamma(\beta)} \int_{-\infty}^{t}(t-\bar{t})^{\beta-1} T_{y}(z, \bar{t}) \mathrm{d} \bar{t},
\end{aligned}
$$

under the following constitutive boundary conditions

$$
\begin{cases}\partial_{z} \chi_{x}(0, t)=\frac{1}{l_{\lambda}} \chi_{x}(0, t), & \partial_{z} \gamma(0, t)=\frac{1}{l_{\lambda}} \gamma(0, t), \\ \partial_{z} \chi_{x}(L, t)=-\frac{1}{l_{\lambda}} \chi_{x}(L, t), & \partial_{z} \gamma(L, t)=-\frac{1}{l_{\lambda}} \gamma(L, t) .\end{cases}
$$

Moreover, taking into account the fractional stress-strain relation in Equations (13) and (27) and boundary conditions in Equation (28) can be rewritten as

$$
\begin{gathered}
\partial_{t^{+}}^{\beta} \partial_{z}^{2} \chi_{x}(z, t)-\frac{1}{l_{\lambda}^{2}} \partial_{t^{+}}^{\beta} \chi_{x}(z, t)=-\frac{M_{x}(z, t)}{l_{\lambda}^{2} \mathrm{E}_{\beta} \mathcal{I}_{x}}, \\
\partial_{t^{+}}^{\beta} \partial_{z}^{2} \gamma(z, t)-\frac{1}{l_{\lambda}^{2}} \partial_{t^{+}}^{\beta} \gamma(z, t)=-\frac{T_{y}(z, t)}{l_{\lambda}^{2} G_{\beta} A_{s}}, \\
\left\{\begin{array}{l}
\partial_{t^{+}}^{\beta} \partial_{z} \chi_{x}(0, t)=\frac{1}{l_{\lambda}} \partial_{t^{+}}^{\beta} \chi_{x}(0, t), \quad \partial_{t^{+}}^{\beta} \partial_{z} \gamma(0, t)=\frac{\partial_{t^{+}}^{\beta} \gamma(0, t)}{l_{\lambda}}, \\
\partial_{t^{+}}^{\beta} \partial_{z} \chi_{x}(L, t)=-\frac{1}{l_{\lambda}} \partial_{t^{+}}^{\beta} \chi_{x}(L, t), \quad \partial_{t^{+}}^{\beta} \partial_{z} \gamma(L, t)=-\frac{\partial_{t^{+}}^{\beta} \gamma(L, t)}{l_{\lambda}},
\end{array}\right.
\end{gathered}
$$

where $\partial_{t^{+}}^{\beta}$ denotes the partial counterpart of the fractional differential operator in Equation (14). Equation (29) represent the viscoelastic nonlocal beam problem where the time-dependent behaviour is modeled by a fractional-order law and space nonlocal behaviour is expressed by stress-driven approach.

The kinematic relation in Equation (21) leads to the following relation in terms of curvature

$$
\chi_{x}(z, t)=\partial_{z} \varphi_{x}(z, t)=\partial_{z} \gamma(z, t)-\partial_{z}^{2} v(z, t),
$$

the nonlocal relations in Equation (29a) yields

$$
\partial_{t^{+}}^{\beta}\left[\partial_{z}^{3} \gamma(z, t)-\partial_{z}^{4} v(z, t)-\frac{\partial_{z} \gamma(z, t)-\partial_{z}^{2} v(z, t)}{l_{\lambda}^{2}}\right]=-\frac{M_{x}(z, t)}{l_{\lambda}^{2} \mathrm{E}_{\beta} \mathcal{I}_{x}}
$$

and taking into account equilibrium equation in Equations (18a) and (20), from Equations (29b) and (31) we get

$$
\partial_{t^{+}}^{\beta}\left[\partial_{z}^{5} \gamma(z, t)-\partial_{z}^{6} v(z, t)-\frac{\partial_{z}^{3} \gamma(z, t)-\partial_{z}^{4} v(z, t)}{l_{\lambda}^{2}}\right]=\frac{q_{y}(z, t)+\partial_{z} m(z, t)}{l_{\lambda}^{2} \mathrm{E}_{\beta} \mathcal{I}_{x}},
$$




$$
\partial_{t^{+}}^{\beta} \partial_{z}^{5} \gamma(z, t)=\frac{1}{l_{\lambda}^{2}} \partial_{t^{+}}^{\beta} \partial_{z}^{3} \gamma(z, t)+\frac{\partial_{z}^{2} q_{y}(z, t)}{l_{\lambda}^{2} \mathrm{G}_{\beta} A_{s}},
$$

and by combining the two equations we obtain

$$
\partial_{t^{+}}^{\beta}\left[-\partial_{z}^{6} v(z, t)+\frac{\partial_{z}^{4} v(z, t)}{l_{\lambda}^{2}}\right]=\frac{q_{y}(z, t)+\partial_{z} m(z, t)}{l_{\lambda}^{2} \mathrm{E}_{\beta} \mathcal{I}_{x}}-\frac{\partial_{z}^{2} q_{y}(z, t)}{l_{\lambda}^{2} \mathrm{G}_{\beta} A_{s}},
$$

and the constitutive boundary conditions in Equation (29c) can be rewritten taking into account kinematic relation in Equation (30). We can observe that when the nonlocal parameter $\lambda \rightarrow 0 \Rightarrow l_{\lambda} \rightarrow 0$ and then Equation (33) becomes

$$
\partial_{t^{+}}^{\beta} \partial_{z}^{4} v(z, t)=\frac{q_{y}(z, t)+\partial_{z} m(z, t)}{\mathrm{E}_{\beta} \mathcal{I}_{x}}-\frac{\partial_{z}^{2} q_{y}(z, t)}{\mathrm{G}_{\beta} A_{s}},
$$

that is the fractional differential equation of the viscoelastic Timoshenko beam [47].

\section{Sample Applications: Simply Supported Viscoelastic Micro-Beam}

In the example reported below, the effects of the nonlocal parameter $\lambda$ and viscoelastic parameter $\beta$ in the mechanical response are highlighted. The considered case represents a mechanical model of a MEMS resonator [48]. Specifically, a simply supported microbeam of length $L=300 \mu \mathrm{m}$ and a rectangular cross-section $A=30 \times 25 \mu \mathrm{m}^{2}$, forced by a transversal uniform distributed load, is considered. The load is constant during the time for $t \geqslant 0$, then, it is modeled by means of a unit step function. That is,

$$
q_{y}(z, t)=a U(t),
$$

where $a=10^{-3} \mathrm{mN}$ and $U(t)$ is the Heaviside step function.

MEMS are usually made of piezoresistive nanocomposites, where the matrix is a synthetic resin filled by nanofibers [49-51]. In this sample application, epoxy resin is used as matrix of MEMS and, due to its rheological properties, the micro-beam at hand shows viscoelastic behaviour. The fractional-order law in Equation (16), with $\beta=0.04$ and $C_{\beta}=2660 \mathrm{MPa} \mathrm{s}^{\beta}$, is exploited to model the stress-strain relation of the epoxy resin [39].

The mechanical model takes into account the viscoelastic properties due to the polymeric matrix of the micro-beam and the nonlocal phenomena related to size effects at small scales. The size effect influences the mechanical response in the space, that is simulated by the stress-driven approach described above. The responses in terms of displacement, rotation and curvature for different values of nonlocal parameter $\lambda$ are reported to show the scale phenomenon.

By taking into account load condition in Equation (35) and the definition of fractional integration in Equation (17), Equation (32a) yields

$$
-\partial_{z}^{6} v(z, t)+\frac{\partial_{z}^{4} v(z, t)}{l_{\lambda}^{2}}=\frac{a}{l_{\lambda}^{2} \mathrm{E}_{\beta} \mathcal{I}_{x} \Gamma(\beta)} \int_{0}^{t}(t-\bar{t})^{\beta-1} \mathrm{~d} \bar{t}=\frac{a t^{\beta}}{l_{\lambda}^{2} \mathrm{E}_{\beta} \mathcal{I}_{x} \Gamma(\beta)},
$$

the constitutive boundary conditions are the same in Equation (29c), the kinematic and the static ones are

$$
\begin{aligned}
& v(0, t)=0, \quad v(L, t)=0, \\
& M(0, t)=0, \quad M(L, t)=0 .
\end{aligned}
$$

A solution of this bending problem for an assigned nonlocal parameter $\lambda$ is depicted in Figure 2. Specifically, that picture shows the nonlocal viscoelastic response of a simply supported Timoshenko micro-beam in epoxy resin for different time steps in Figure 2a and the evolution of the displacement at mid-point in Figure 2b. From these figures it can be observed that the viscoelasticity leads to a time-dependent behaviour of the structural element. Moreover, the considered stress-strain relation provides also a size effect by 
the nonlocal parameter $\lambda$. The influence of $\lambda$ in the mechanical response is described subsequently introducing a detailed parametric study.

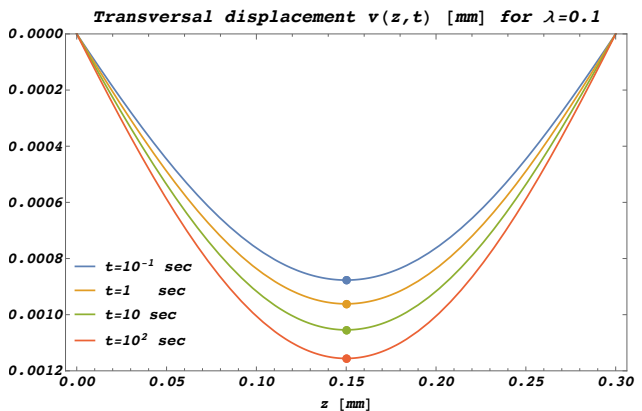

(a)

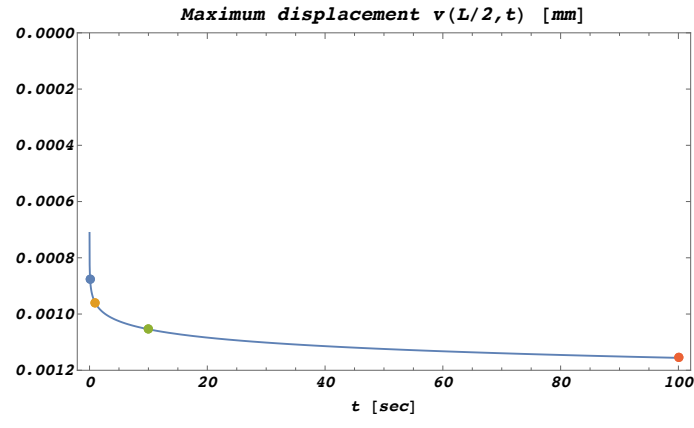

(b)

Figure 2. Displacements of nonlocal micro-beam in epoxy resin with $\lambda=0.1$. (a) Vertical displacement for different time step; (b) Time evolution of displacement at the mid-point.

By virtue of the correspondence principle, the viscoelastic nonlocal response is obtained as an evolution in time of the nonlocal elastic response. For instance, the response in terms of displacement shown in Figure 2 can be seen as a modulating function, the time-varying function $g(t)$, which multiplies the nonlocal elastic space-dependent response $\Phi(z)$. That is,

$$
v(z, t)=\Phi(z) g(t),
$$

for the considered application, the time-dependent function is a power-law function of order $g(t) \propto t^{\beta}$. The space-dependent part can be defined as a shape function.

\section{Influence of the Nonlocal Parameter $\lambda$}

In the proposed formulation, the size effect is ruled by the nonlocal parameter $\lambda$. Its influence on the mechanical response of the considered simply supported Timoshenko micro-beam is shown below, providing a parametric study. Specifically, by varying the nonlocal parameter, three kinematics entities are reported. That is, vertical displacement $v(z, t)$, rotation $\varphi(z, t)$ and bending curvature $\chi_{x}(z, t)$, for different values of $\lambda$.

Figure 3 shows the response in terms of transversal displacement $v(z, t)$ of the cantilever micro-beam made of epoxy resin for different values of nonlocal parameter $\lambda$. Specifically, the time-evolution of the transversal displacement of the longitudinal axis for $\lambda=\{0.1,0.2,0.3,0.4,0.5\}$ is depicted.

Whereas, for the same values of nonlocal parameters, the time-dependent cross-section rotation $\varphi_{x}(z, t)$ and bending curvature $\chi_{x}(z, t)$ are reported in Figure 4.

Figure 3 shows that when the nonlocal parameter increases, the displacement decreases. This fact is also evident in Figure 5 where the time evolution of maximum displacement $v(L / 2, t)$ is depicted for different values of $\lambda$.

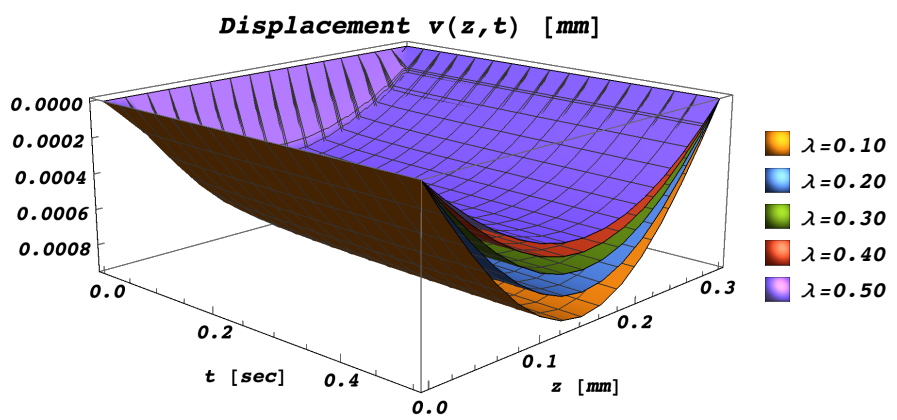

Figure 3. Displacement for different values of $\lambda$. 

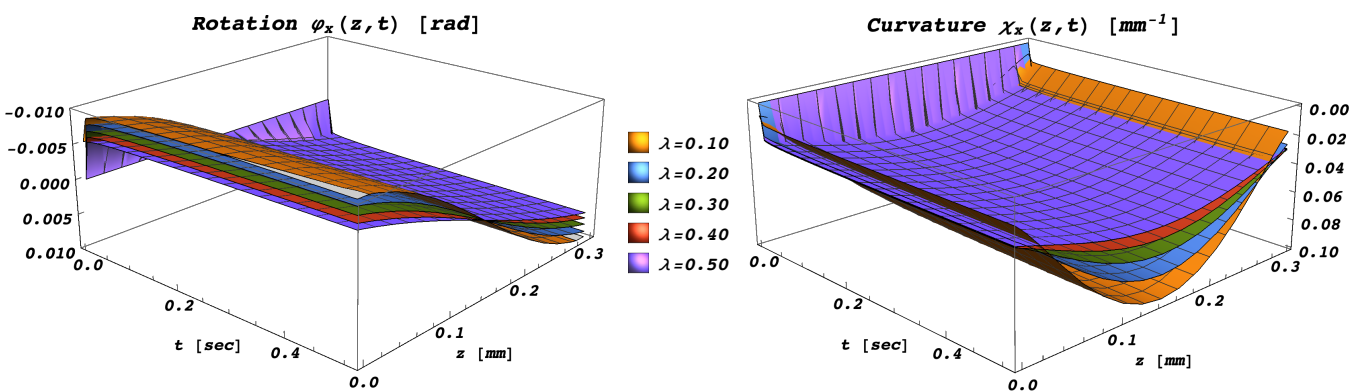

Figure 4. Rotation and curvature for different values of $\lambda$.

Moreover, in order to highlight and isolate the nonlocal effect on the structural response, we compare the kinematic entities at an assigned time. Nonlocality yields a space variation in the shape function, so the time assumption does not imply any loss of generality in the shown results to understand its effects on the mechanical response. Specifically, Figure 6 shows the displacements for different values of $\lambda$ at $t=1 \mathrm{~s}$.

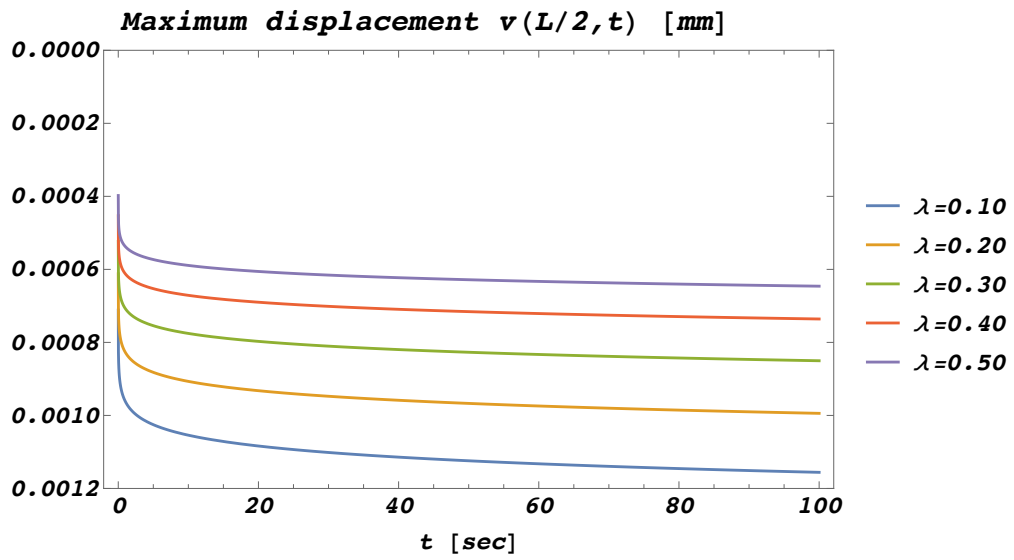

Figure 5. Time evolution of the vertical displacement at the mid-point for different values of $\lambda$.

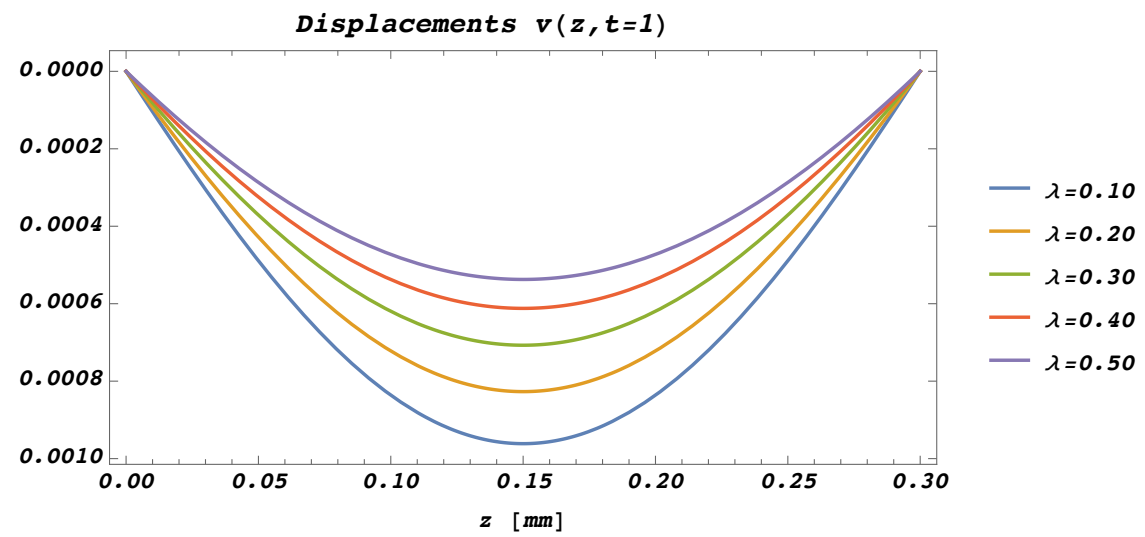

Figure 6. Vertical displcaments for different values of $\lambda$ at $t=1 \mathrm{~s}$.

Whereas, the trends in the function of the nonlocal parameter for the other two kinematics entities are reported in Figure 7. 


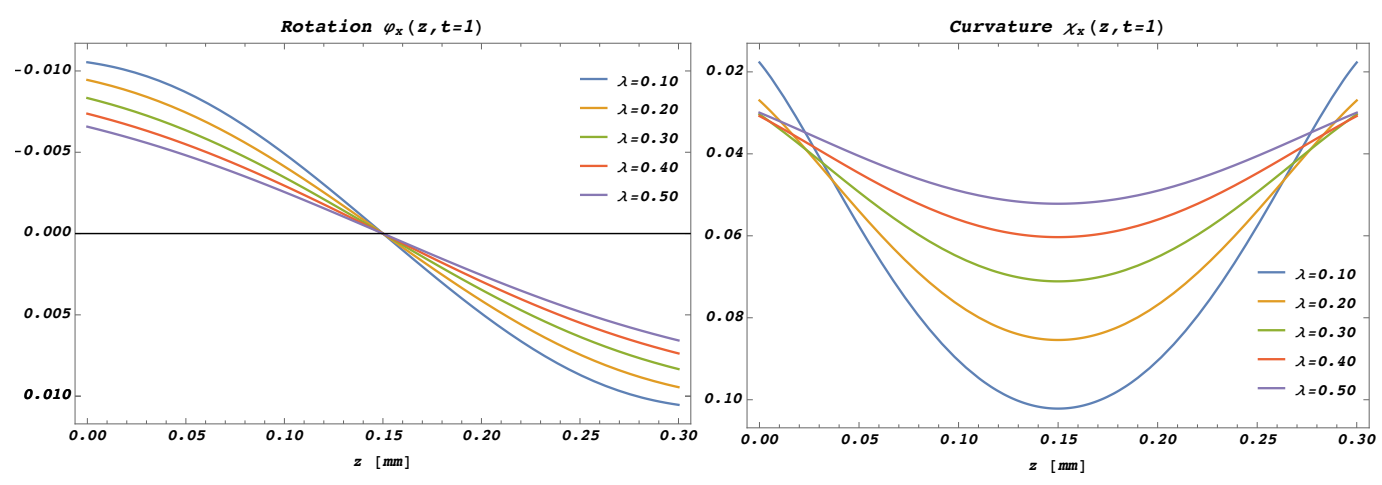

Figure 7. Rotation and curvature for different values of $\lambda$ at $t=1 \mathrm{~s}$.

All these Figures show that when $\lambda$ increases the magnitude of displacement and rotation decrease. Whereas, the nonlocal bending curvature is characterized by a different behaviour. Specifically, its maximum value at $z=L / 2$ decreases when $\lambda$ increases and the space distribution changes. This behaviour can be explained taking into account that there is an increment of long-range interactions when $\lambda$ grows up.

\section{Concluding Remarks}

Bending behaviour modeling is a crucial issue in the design of several structures at small scales. In this context, a Timoshenko bending problem of a nonlocal viscoelastic beam has been investigated and some analytical solutions have been provided. Specifically, in the presented work two main phenomena have been considered that occur in several small-scale structures, that is, time-dependent behaviour and size effects.

Time-dependent behaviour has been obtained with the aid of classical linear viscoelasticity, Boltzmann superposition integral with power-law kernel has been used to provide a time dependent stress-strain relation. The kernel assumption implies that fractional-order operator appears in the constitutive law. In this way, the memory effects are related to the fractional-order $0 \leqslant \beta \leqslant 1$. This particular choice allows good results in the simulation of the real hereditary mechanical behaviour by means of a proper selection of few numbers of parameters.

Stress-driven integral theory has been used to simulate nonlocal effects. This formulation avoids paradoxical problems of Eringen's strain-driven model, consists in a well-posed integral nonlocal relation and provides some useful analytical solutions. By the proposed approach space nonlocality is ruled by a parameter $\lambda$ which weights long-range interactions in the stress-strain relation.

Both phenomena are of interest in the modeling of structures at small scales. Their relative models can be seen as nonlocal formulations. Specifically,

- The Boltzmann superposition integral produces a time-dependent nonlocality, where states of the past influence the mechanical response in the present and the future. For this reason, it is used to call this characteristic memory and hereditary materials those elements that exhibit this behaviour.

- $\quad$ Stress-driven integral theory yields a space-dependent nonlocality, in this case there is a long-range influence between points of the continuum in different positions, and the strain at any point of the medium depends nonlocally on the stress field.

Combining time-dependent Boltzmann and space-dependent Fredholm integrals and introducing Timoshenko's kinematics assumptions for the beam, the analytical solution of viscoelastic nonlocal beam problem has been provided and discussed. Some closedform expressions have been detected and a detailed parametric study has been provided discussing the influences of the nonlocal parameter $\lambda$ on the mechanical response of micro-beams in epoxy resin. The provided analytical solutions, the capabilities of the proposed model to simulate hereditariness and nonlocality in small-scale structures, related 
parametric study and the contributed outcomes presented in this can be useful in the design and optimization of sensors at the micro- and nanoscales.

Author Contributions: Conceptualisation, methodology, software, validation, investigation, writingreview and editing: F.P.P., R.B., F.M.d.S. and A.P. All the authors contributed equally to this work. All authors have read and agreed to the published version of the manuscript.

Funding: This research received no external funding.

Institutional Review Board Statement: Not applicable.

Informed Consent Statement: Not applicable.

Data Availability Statement: The data presented in this study are available within this article. Further inquiries may be directed to the authors.

Acknowledgments: The authors gratefully acknowledge the support received from the MIUR in the framework of the Project PRIN 2017 code 2017J4EAYB Multiscale Innovative Materials and StructuresMIMS (University of Naples Federico II and University of Palermo Research Units). Francesco Marotti de Sciarra gratefully acknowledges MIUR in the framework of the Project PRIN 2020 code 20209F3A37 Sustainable modelling of materials, structures and urban spaces including economic-legal implications (University of Padova Research Unit).

Conflicts of Interest: The authors declare no conflict of interest.

\section{Abbreviations}

The following abbreviations are used in this manuscript:

MEMS micro electromechanical system

NEMS nano electromechanical system

\section{References}

1. Mojahedi, M. Size dependent dynamic behaviour of electrostatically actuated microbridges. Int. J. Eng. Sci. 2017, 111, 74-85. [CrossRef]

2. De Bellis, M.L.; Bacigalupo, A.; Zavarise, G. Characterization of hybrid piezoelectric nanogenerators through asymptotic homogenization. Comput. Methods Appl. Mech. Eng. 2019, 355, 1148-1186. [CrossRef]

3. Kiani, K.; Zur, K.K. Vibrations of double-nanorod-systems with defects using nonlocal-integral-surface energy-based formulations. Compos. Struct. 2021, 256, 113028. [CrossRef]

4. Malikan, M.; Uglov, N.S.; Eremeyev, V.A. On instabilities and post-buckling of piezomagnetic and flexomagnetic nanostructures Int. J. Eng. Sci. 2020, 157, 103395. [CrossRef]

5. Luciano, R.; Willis, J.R. Boundary-layer corrections for stress and strain fields in randomly heterogeneous materials. J. Mech. Phys. Solids 2003, 51, 1075-1088. [CrossRef]

6. Rahmani, O.; Pedram, O. Analysis and modeling the size effect on vibration of functionally graded nanobeams based on nonlocal Timoshenko beam theory. Int. J. Eng. Sci. 2014, 77, 55-70. [CrossRef]

7. Fuschi, P.; Pisano, A.A.; Polizzotto, C. Size effects of small-scale beams in bending addressed with a strain-difference based nonlocal elasticity theory. Int. J. Mech. Sci. 2019, 151, 661-671. [CrossRef]

8. Jankowski, P.; Zur, K.K.; Kim, J.; Reddy, J.N. On the bifurcation buckling and vibration of porous nanobeams. Compos. Struct. 2020, 250, 112632. [CrossRef]

9. Malikan, M.; Eremeyev, V.A.; Zur, K.K. Effect of axial porosities on flexomagnetic response of in-plane compressed piezomagnetic nanobeams. Symmetry 2020, 12, 1935. [CrossRef]

10. Vaccaro, M.S.; Pinnola, F.P.; de Sciarra, F.M.; Canadija, M.; Barretta, R. Stress-driven two-phase integral elasticity for Timoshenko curved beams. Proc. Inst. Mech. Eng. Part J. Nanomater. Nanoeng. Nanosyst. 2021, 235, 52-63. [CrossRef]

11. Di Matteo, A.; Pavone, M.; Pirrotta, A. Exact and Approximate Analytical Solutions for Nonlocal Nanoplates of Arbitrary Shapes in Bending Using the Line Element-Less Method. Meccanica 2021, 1-19. [CrossRef]

12. Rogula, D. Influence of spatial acoustic dispersion on dynamical properties of dislocations. Bull. Acad. Pol. Sci. Ser. Sci. Tech. 1965, 13, 337-343.

13. Rogula, D. Introduction to nonlocal theory of material media. In Nonlocal Theory of Material Media; CISM Courses and Lectures; Rogula, D., Ed.; Springer: Wien, Austria, 1982; Volume 268, pp. 125-222.

14. Flugge, W. Viscoelasticity; Blaisdell Publishing Company: Waltham, MA, USA, 1967.

15. Christensen, R.M. Theory of Viscoelasticity, an Introduction; Academic Press: New York, NY, USA, 1982.

16. Tricomi, F.G. Integral Equations; Interscience: New York, NY, USA, 1957. 
17. Mainardi, F. Fractional Calculus and Waves in Linear Viscoelasticity; Imperial College: London, UK, 2010.

18. Luciano, R.; Willis, J.R. Non-local constitutive response of a random laminate subjected to configuration-dependent body force. J. Mech. Phys. Solids 2001, 49, 431-444. [CrossRef]

19. Lam, D.C.C.; Yang, F.; Chong, A.C.M.; Wang, J.; Tong, P. Experiments and theory in strain gradient elasticity. J. Mech. Phys. Solids 2003, 51, 1477-1508. [CrossRef]

20. Romano, G.; Diaco, M.; On formulation of nonlocal elasticity problems. Meccanica 2020, 56, 1303-1328. [CrossRef]

21. Lim, C.W.; Zhang, G.; Reddy, J.N. A higher-order nonlocal elasticity and strain gradient theory and its applications in wave propagation. J. Mech. Phys. Solids 2015, 78, 298-313. [CrossRef]

22. Barretta, R.; de Sciarra, F.M. Constitutive boundary conditions for nonlocal strain gradient elastic nano-beams. Int. J. Eng. Sci. 2018, 130, 187-198. [CrossRef]

23. Di Paola, M.; Pirrotta, A.; Zingales, M. Mechanically-based approach to non-local elasticity: Variational principles. Int. J. Solids Struct. 2010, 47, 539-548. [CrossRef]

24. Di Paola, M.; Failla, G.; Zingales, M. Non-local stiffness and damping models for shear-deformable beams. Eur. J. Mech. A/Solids 2013, 40, 69-83. [CrossRef]

25. Zur, K.K.; Arefi, M.; Kim, J.; Reddy, J.N. Free vibration and buckling analyses of magneto-electro-elastic FGM nanoplates based on nonlocal modified higher-order sinusoidal shear deformation theory. Compos. Part B Eng. 2020, 182, 107601. [CrossRef]

26. Eringen, A.C. Linear theory of nonlocal elasticity and dispersion of plane waves. Int. J. Eng. Sci. 1972, 10, 425-435. [CrossRef]

27. Eringen, A.C. On differential equations of nonlocal elasticity and solutions of screw dislocation and surface waves. J. Appl. Phys. 1983, 54, 4703. [CrossRef]

28. Challamel, N.; Wang, C.M. The small length scale effect for a non-local cantilever beam: A paradox solved. Nanotechnology 2008, 19, 345703. [CrossRef]

29. Fernández-Sáez, J.; Zaera, R.; Loya, J.A.; Reddy, J.N. Bending of Euler-Bernoulli beams using Eringen's integral formulation: A paradox resolved. Int. J. Eng. Sci. 2016, 99, 107-116. [CrossRef]

30. Romano, G.; Barretta, R.; Diaco, M.; de Sciarra, F.M. Constitutive boundary conditions and paradoxes in nonlocal elastic nano-beams. Int. J. Mech. Sci. 2017, 121, 151-156. [CrossRef]

31. Romano, G.; Barretta, R. Nonlocal elasticity in nanobeams: The stress-driven integral model. Int. J. Eng. Sci. 2017, 115, 14-27. [CrossRef]

32. Romano, G.; Barretta, R. Stress-driven versus strain-driven nonlocal integral model for elastic nano-beams. Compos. Part B Eng. 2017, 114, 184-188. [CrossRef]

33. Barretta, R.; Fabbrocino, F.; Luciano, R.; de Sciarra, F.M.; Ruta, G. Buckling loads of nano-beams in stress-driven nonlocal elasticity. Mech. Adv. Mater. Struct. 2020, 27, 869-875. [CrossRef]

34. Nutting, P.G. A new general law of deformation. J. Frankl. Inst. 1921, 191, 679-685. [CrossRef]

35. Bagley, R.L.; Torvik, P.J. On the appearance of the fractional derivative in the behavior of real materials. J. Appl. Mech. 1984, 51, 294-298.

36. Di Paola, M.; Pirrotta, A.; Valenza, A. Visco-elastic behaviour through fractional calculus: An easier method for best fitting experimental results. Mech. Mater. 2011, 43, 799-806. [CrossRef]

37. Celauro, C.; Fecarotti, C.; Pirrotta, A.; Collop, A.C. Experimental validation of a fractional model for creep/recovery testing of asphalt mixtures. Constr. Build. Mater. 2012, 36, 458-466. [CrossRef]

38. Demirci, N.; Tonuk, E. Non-integer viscoelastic constitutive law to model soft biological tissues to in-vivo indentation. Acta Bioeng. Biomech. 2014, 16, 13-21. [PubMed]

39. Di Paola, M.; Fiore, V.; Pinnola, F.P.; Valenza, A. On the influence of the initial ramp for a correct definition of the parameters of the fractional viscoelastic material. Mech. Mater. 2014, 69, 63-70. [CrossRef]

40. Alotta, G.; Di Paola, M.; Pirrotta, A. Fractional Tajimi-Kanai model for simulating earthquake ground motion. Bull. Earthq. Eng. 2014, 12, 2495-2506. [CrossRef]

41. Podlubny, I. Fractional Differential Equation; Academic Press: San Diego, CA, USA, 1999.

42. Hilfer, R. Application of Fractional Calculus in Physics; World Scientific: Singapore, 2000.

43. Gemant, A. A method of analyzing experimental results obtained from elasto-viscous bodies. Physics 1936, 7, 311-317. [CrossRef]

44. Scott Blair, G.W.; Caffyn, J.E. An application of the theory of quasi-properties to the treatment of anomalous strain? stress relations. Philos. Mag. 1949, 40, 80-94. [CrossRef]

45. Slonimsky, G.L. On the law of deformation of highly elastic polymeric bodies. Dokl. Akad. Nauk SSSR 1961, 140, 343-346. (In Russian)

46. Di Matteo, A.; Pirrotta, A. Generalized Differential Transform Method for Nonlinear Boundary Value Problem of Fractional Order. Commun. Nonlinear Sci. Numer. Simul. 2015, 29, 88-101. [CrossRef]

47. Pirrotta, A.; Cutrona, S.; Di Lorenzo, S.; Di Matteo, A. Fractional visco-elastic Timoshenko beam deflection via single equation. Int. J. Numer. Methods Eng. 2015, 104, 869-886. [CrossRef]

48. Goeders, K.M.; Colton, J.S.; Bottomley, L.A. Microcantilevers: Sensing chemical interactions via mechanical motion. Chem. Rev. 2008, 108, 522-542. [CrossRef]

49. Luciano, R.; Willis, J.R. FE analysis of stress and strain fields in finite random composite bodies. J. Mech. Phys. Solids 2005, 53, 1505-1522. [CrossRef] 
50. Tang, G.; Liu, J.-Q.; Liu, H.-S.; Li, Y.-G.; Yang, C.-S.; He, D.-N.; Dzungdao, V.; Tanaka, K.; Sugiyama, S. Piezoelectric MEMS generator based on the bulk PZT/silicon wafer bonding technique. Phys. Status Solidi (A) Appl. Mater. Sci. 2011, 208, 2913-2919. [CrossRef]

51. Acierno, S.; Barretta, R.; Luciano, R.; de Sciarra, F.M.; Russo, P. Experimental evaluations and modeling of the tensile behavior of polypropylene/single-walled carbon nanotubes fibers. Compos. Struct. 2017, 174, 12-18. [CrossRef] 